\title{
An Efficient Algorithm for Removal of Salt and Pepper Noise from Images
}

\author{
Chetana Kankariya \\ PG Student \\ Dept. of EC \\ TIT COE, Bhopal
}

\author{
Sudesh Gupta \\ Asst. Professor \\ Dept. of Electronics \\ TIT COE, Bhopal
}

\begin{abstract}
:
Noise deletion is a significant issue in the field of image processing. In this paper switching threshold algorithm for the elimination of high density salt and pepper noise is proposed. It is implemented in two steps; firstly switching threshold is applied to the whole image to detect the pixels as corrupted/uncorrupted. Secondly, new pixel value is anticipated only for the corrupted pixels. The algorithm use an adaptive length window having maximum size is of $7 \times 7$. The existing methods are Arithmetic Mean Filtering (AMF) technique, Geometric Mean Filtering (GMF) technique, Harmonic Mean Filtering (HMF) technique. The proposed algorithm automatically switches the window size as per the noise occurrence. Performance of algorithm is evaluated in terms of Mean square Error, Peak Signal to Noise Ratio, Image Enhancement Factor, and processing time and evaluated with other filtering techniques. Extensive simulation shows that proposed algorithm removes the noise even though noise level as high as $50 \%$ and produces better results than that of existing filtering techniques.
\end{abstract}

\section{Keywords:}

Impulse noise; Adaptive median filter; Peak signal to noise ratio (PSNR).

\section{INTRODUCTION}

The image processing field has broad uses. Noise is any unwanted information that contaminates an image. Images are contaminated by noise due to faults generated in sensors or communication medium. Noise should be eliminated in the images before identification, segmentation and detection [2]. Noise appears in an image from diverse sources of Digital image are sometime affected by unlike types of noise, specifically, additive white Gaussian noise, impulse noise and mixed impulse noise [4].The Salt \& Pepper type noise is mainly caused by unsatisfactory work of the pixel elements in the camera sensors, improper storage locations or timing errors. For the images despoiled by Salt \& Pepper noise, the noisy pixels can take solitary maximum and minimum value in the dynamic range [1].

A variety of filtering procedures have been used for removing impulse noise. Image Denoising techniques are applicable to eliminate such random noises without disturbing the important signal features. Firstly, the function of Denoising is to eliminate the noise while retaining the original image details. In gray scale images white and black dots indicate the salt and pepper noise called impulse noise which chaotically scattered along image area [4]. Previously, linear filters in general produce serious image blurring. As an innovation,

Non-linear filters have been broadly exploited for Denoising, due to the characteristics of nonlinear filters like improved filtering performance, impulse noise shrinking and border/details conservation. [5].
Existing mean filtering technique works very simple. For this, single pixel is considered at a time and sub window is considered around that pixel. Then mean is computed using the pixel value of that sub window. Then, the considered pixel is exchanged with that mean. This way, all the mean filtering techniques work.

Then mean is computed using the pixel value of that sub window. Then, the considered pixel is exchanged with that mean. These way all the mean filtering techniques work.

There are some problems associated with the filtering techniques. They are not applicable at higher noise densities. Also, the PSNR obtained by these techniques is lower than that of ruined images. So it decreases the quality of images rather improving image. Thus, the filters with good border and image detail conservation properties are highly advantageous for image filtering. The median filter and its variants are amongst mainly used filters for impulse noise deletion. When median filters are applied uniformly across the image, be likely to alter both noisy as well as noise free pixels, resulting in blurred and indistinct features. Freshly, some modified forms of the median filter have been projected to overcome these constraints. In these variants of the median filter technique, the pixel value is modified only when it is found ruined with noise. These statistics of the median filter still maintains the basic grade structure of the filter. Among these filters, the center weighted median filters (CWMFs) give a huge weight to the middle pixel, while selecting between the current pixel and the median value. In order to pass up the impact of the noisy pixels on the filtered output, it is necessary to execute the impulse detection before filtering. [10]

In this paper simultaneous removal of salt and pepper noise is addressed. But, simply pixel value with 0 and 255 are treated as corrupted, with other values as uncorrupted. Our approach of median filter of adequate window size is used for the recognition of degraded pixel. The complete diversity between the pixel of interest and the median filtered output is obtained and compared with the threshold obtained from the minimum and maximum pixel values in the preferred window. A binary flag image is obtained with its values 1 for the degraded pixels and 0 for the unaffected pixels. The corrupted pixel values are projected for the new values using the median filtering technique.

\section{EXISTING FILTERING TECHNIQUES:}

To get better image from its noise there exist multiple filtering procedures which are application oriented. Some filtering procedures are explained below:

\subsection{Arithmetic Mean Filtering (AMF) technique:}

Working of AMF filter is simplest among all filters. Sxy signifies the set of coordinates in a rectangular sub image window of size $\mathrm{m} \mathrm{X} \mathrm{n}$, centered at point $(\mathrm{x}, \mathrm{y})$. The arithmetic mean filtering practice calculates the average value of the degraded image $g(x$, 
y) in the area define by Sxy. The value of the restore image at any point $(x, y)$ is simply the arithmetic mean calculated with the pixels in the region define by $\mathrm{S}$.

$$
f(x, y) \frac{1}{m n} \sum_{(s, t) \in S_{x y}} g(s, t)
$$

\subsection{Geometric mean filtering (GMF) technique:}

For GMF procedure each restore pixel is given by the creation of the pixels in the sub image window, raised to the power $1 / \mathrm{mn}$. A GMF achieves smooth image but it tends to lose less image quality through the method. GMF can be expressed by the expression given below:

$$
f(x, y)=\left[\prod_{(s, t) \in S_{x y}} g(s, t)\right] \frac{1}{m n}
$$

\subsection{Harmonic Mean Filtering (HMF) Technique:}

The Harmonic Mean Filter works well for Salt noise but fails for Pepper noise. It does well also with some other types of noise like Gaussian noise.

\subsection{Proposed Approach:}

The standard median filters are effective only at low noise densities as they are applied uniformly across the image, modifying pixels that are undisturbed by noise. They often display blurring for large window sizes, or deficient noise suppression for little window sizes. As an enhancement adaptive window size mean filter algorithms are proposed which achieve a high degree of noise suppression and still reserve image sharpness. The absolute disparity between the pixel of interest and the median filtered output is obtained and evaluated with the threshold obtained from the min and max pixel values in the chosen window.

\section{IMPLEMENTATION DETAILS:}

The algorithm is developed in two steps. First is the identification of corrupted pixel in the image, and the second step is the replacement of only corrupted pixels with the estimated values.

\section{A. Detection of corrupted pixels:}

Table 1: MSE, PSNR, IEF and Processing time for AMF and GMF filters at different noise densities for BRAIN image.
The identification is based on the supposition that a corrupted pixel takes a grey value which is different than the neighboring pixels in the filtering window, where unaffected regions in the image have smoothly varying grey levels separate by borders. For identification of affected or unaffected pixel, a binary flag image $b(i, j)$ is build such that $b(i, j)=1$, when the pixel $x(i, j)$ is damage and $b(i, j)=0$ for unaffected pixel.

\section{B. Estimation of New pixel value for corrupted pixel:}

The corrupted pixels are obtained when the binary flag image $b$ $(i, j)$ is 1 . These pixels are replaced by the new estimated values using median filter for lesser and higher noise densities. For illustration purpose, we guess that the corrupted pixels take values $X_{\text {mas }}=255$ and $X_{\min }=0$.

Case 1: If the number of corrupted pixels " $\mathrm{n}$ " in the window is less than or equals to 4 , that is $n \leq 4$, then two dimensional window of size $3 \times 3$ is selected and median operation performed. Case 2: If the number of corrupted pixels " $n$ " in the window is between 5 to 12 , that is, $5 \leq \mathrm{n} \leq 12$, then $5 \times 5$ median filtering is performed and the corrupted value is replaced by the median value.

Case 3: If the number of corrupted pixels " $n$ " in the window is greater than 13 , switch the window automatically to $7 \times 7$. Thus, switching threshold technique is used in proposed method depending on the window size.

\section{Median Filter Implementation:}

The implementation of median filter consists of computing the median of the grey-level values within the square or rectangular filter window surrounding every pixel. For example, let the mark is a $3 \times 3$ and the pixel values in the nine positions. This filter arranges all the grey level values in the ascending order and selects the middle value and replaces the middle value of that median

\section{SIMULATION RESULTS:}

The Proposed algorithm is tested with different variety of greyscale images. The Performance of algorithm is evaluated quantitatively using the measures viz. Mean Square Error (MSE), Peak signal to noise ratio (PSNR in $\mathrm{dB}$ ), Image enhancement factor (IEF) and Processing Time in Seconds. MATLAB 7.0(R14) on a PC equipped with a $2.13 \mathrm{GHz}$ and $2 \mathrm{~GB}$ of RAM memory has been employed for the evaluation of processing time of all filtering techniques. The values are tabulated in table 1 for

\begin{tabular}{|c|c|c|c|c|c|c|c|c|c|}
\hline \multirow[t]{2}{*}{ Sr. No } & \multirow{2}{*}{$\begin{array}{l}\text { Noise } \\
\text { Level }\end{array}$} & \multicolumn{4}{|c|}{$A M F$} & \multicolumn{4}{|c|}{$G M F$} \\
\hline & & $M S E$ & $P S N R$ & $I E F$ & Processing time & $M S E$ & PSNR & $I E F$ & Processing Time \\
\hline 1 & $10 \%$ & 37.9 & 40.66 & 44.71 & 0.028 & 231.57 & 36.30 & 0.46 & 0.39 \\
\hline 2 & $20 \%$ & 185.44 & 36.78 & 20.74 & 0.027 & 737.07 & 33.79 & 0.14 & 0.027 \\
\hline 3 & $30 \%$ & 469.79 & 34.77 & 10.11 & 0.027 & 1390.34 & 32.4 & 0.08 & 1.10 \\
\hline 4 & $40 \%$ & 9570.28 & 33.22 & 6.1 & 0.028 & 2142.13 & 31.4 & 0.05 & 1.51 \\
\hline
\end{tabular}
Lena image. 


\begin{tabular}{|c|c|c|c|c|c|c|c|c|c|}
\hline 5 & $50 \%$ & 1667.19 & 32.02 & 4.21 & 0.025 & 2915.67 & 30.80 & 0.04 & 1.83 \\
\hline \multirow{2}{*}{ Sr. No } & $\begin{array}{c}\text { Noise } \\
\text { Level }\end{array}$ & \multicolumn{9}{|c|}{} & \multicolumn{5}{|c|}{ Proposed System } \\
\cline { 2 - 10 } & & MSE & PSNR & IEF & $\begin{array}{c}\text { Processing } \\
\text { Time }\end{array}$ & MSE & PSNR & IEF & $\begin{array}{c}\text { Processing } \\
\text { Time }\end{array}$ \\
\hline 1 & $10 \%$ & 229.57 & 36.3 & 0.44 & 0.007 & 4.054 & 45.09 & 343.39 & 0.107 \\
\hline 2 & $20 \%$ & 726.99 & 33.82 & 0.13 & 0.019 & 10.88 & 42.94 & 212.63 & 0.245 \\
\hline 3 & $30 \%$ & 1367.74 & 32.4 & 0.08 & 0.037 & 23.39 & 33.22 & 37.63 & 0.225 \\
\hline 4 & $40 \%$ & 2103.15 & 31.51 & 0.057 & 0.066 & 54.53 & 39.44 & 23.06 & 0.267 \\
\hline 5 & $50 \%$ & 2857.15 & 30.85 & 0.04 & 0.105 & 176.58 & 36.89 & 18.17 & 0.314 \\
\hline
\end{tabular}

Table 2: MSE, PSNR, IEF and Processing time for HMF and Proposed system at different noise densities for BRAIN image.

The obtained results are compared with outputs of all filtering techniques with a proper window size. The value in the table shows that proposed algorithm gives better performance even for high density impulse noise of $50 \%$. The table displays proposed approach gives high PSNR and IEF values. Following figures display subjective visual perception. The proposed algorithm

$$
I E F=\frac{\sum_{i}^{M 1} \sum_{j}^{M 2}[n(i, j)-s(i, j)] 2}{\sum_{i}^{M 1} \sum_{j}^{M 2}[y(i, j)-s(i, j)] 2}
$$

removes salt and pepper noise simultaneously with edge preservation and reduced blurring. It has better subjective quality when compared with all filtering techniques.

$\mathrm{PSNR}=20 \times \log _{10}(255 / \sqrt{\mathrm{MSE}})$

$$
M S E=\frac{\sum_{i}^{M 1} \sum_{j}^{M 2}[y(i, j)-s(i, j)] 2}{M 1 X M 2}
$$

\section{RESULTS FOR BRAIN IMAGE:}
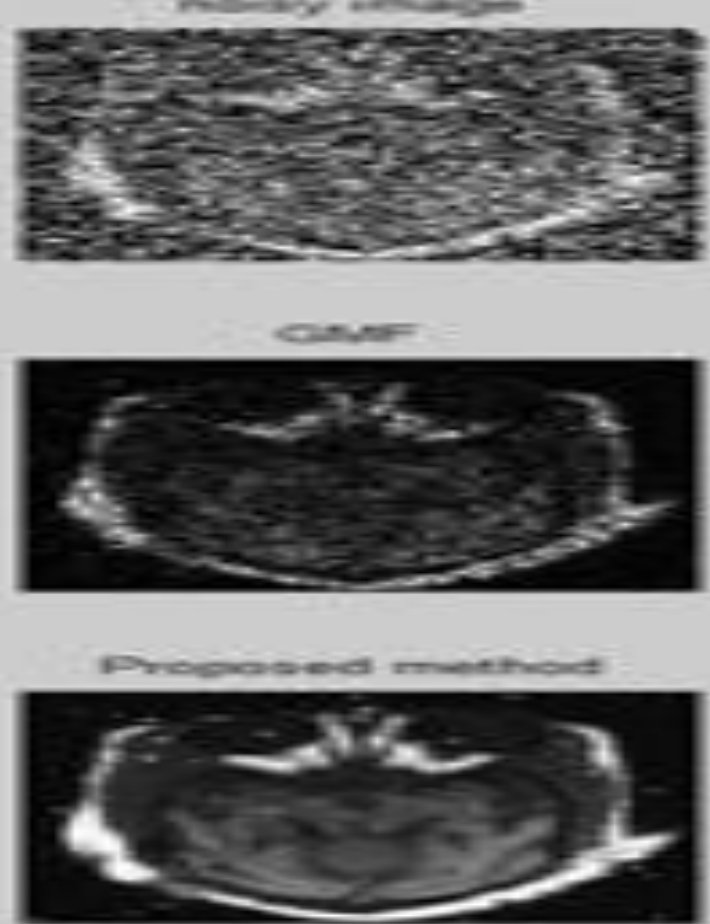


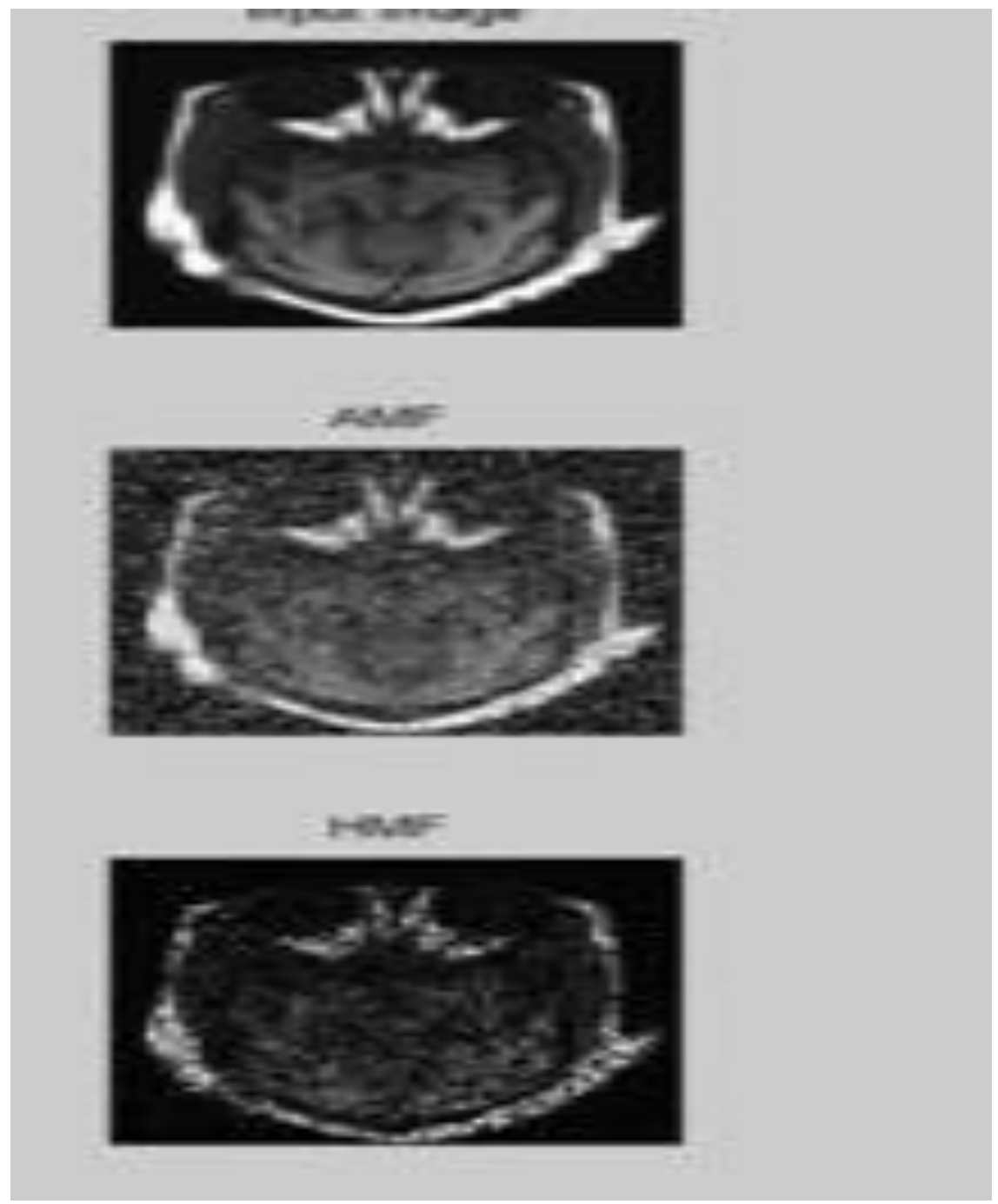

Figure 1. Experimental results for image

\section{CONCLUSION:}

An Improved switching technique for Denoising of high density salt and pepper noise with the help of binary flag in gray scale images is implemented If the pixels are un-affected they are left unaltered, processing is done only if the pixels are corrupted in the second stage. New pixel value is computed using median filter depending on the number of corrupted pixels. The performance of the algorithm is analyzed using MSE, PSNR, IEF and processing time. The obtained results are compared with other filtering techniques. The simulation outcome shows the proposed algorithm that gives better performance as compare to other filtering procedures.

\section{REFERENCES:}

[1] Kaisar S., Md. Sakib Rijwan Jubayer Al Mahmud Muhammad "Salt and Pepper Noise Detection and removal by Tolerance based Selective Arithmetic Mean Filtering Technique for image restoration" in IJCSNS International Journal of Computer Science and Network Security, VOL.8 No.6pp 271-278, June 2008.

[2] Zongwei Lu, Zhide Tang, Lin Zhou, Hao Yang and Lisen Lin "One Simple and Fast Method for the Efficient Removal of High - Density Salt and Pepper Impulse Noise" in 2nd International Conference on Information and Multimedia Technology (ICIMT 2010) IPCSIT vol. 42 (2012) () (2012) IACSIT Press, Singapore

[3] Rama S., tulasi P.Shiva Prasad "A new efficient algorithm for removing of high density salt and pepper noise through modified median filter for video restoration"

[4] Ansari Mohd Dilshad, Garima Singh, Arjun Singh, Ashwani Kumar "An Efficient Salt and Pepper noise Removal and Edge preserving Scheme for Image Restoration" in Int. J. Computer Technology \& Applications,Vol 3 (5), 1848-1854

[5] Juneja Mamta, Rajni Mohana "An Improved Adaptive Median Filtering Method for Impulse Noise Detection" in International Journal of Recent Trends in Engineering, Vol 1, No. 1, May 2009.

[6] Chun-mei WANG and Tian-yi LI "An Improved Adaptive Median Filter for Image Denoising" in 3rd International Conference on Computer and Electrical Engineering (ICCEE 2010)IPCSIT vol. 53 (2012) (C) (2012) IACSIT Press, SingaporeDOI: 10.7763/IPCSIT.2012.V53.No.2.64

[7] Manikandan S. and D. Ebenezer, "A Nonlinear DecisionBased Algorithm for Removal of Strip Lines, Drop Lines, Blotches, Band Missing and Impulses in Images and 
Videos" EURASIP Journal on Image and Video Processing, Hindawi Publishing Corporation, Volume 2008, pp 1-10

[8] Pandey Rajoo, “An Improved Switching Median filter for Uniformly Distributed Impulse Noise Removal", World Academy of Science, Engineering and Technology, 2008

[9] Kornatowski Eugeniusz - Krzysztof Okarma, "Probabilistic Measure Of Colour Image Processing Fidelity", Journal of Electrical Engineering, Vol. 59, pp.2933, No. 1, 2008

[10] Gnanadurai D., and V.Sadasivam, "An Efficient Adaptive Thresholding Technique for Wavelet Based Image
Devoicing”. International Journal of Signal Processing, Vol.2, pp.115-128, no. 2, 2003

[11] R Abhishek, Srinivas N "An Advanced Technique for Removal Of Salt \& Pepper Noise In Images" in International Journal Of Engineering And Computer Science ISSN:2319-7242 Volume 2 Issue 9 September 2013 Page No. 2856-2860 Abhishek R, IJECS Volume 2 Issue 9 September, 2013 Page No. 2856-2860 Page 2856

[12] Gonzales R. C. and R. E. Woods, "Digital Image Processing" 2nd ed. Reading, MA: Addison -Wesley, 2002. 